\title{
Negative results on acyclic improper colorings
}

\author{
Pascal Ochem ${ }^{1 \dagger}$ \\ ${ }^{1}$ LaBRI, Université Bordeaux I \\ 351, cours de la Libération, 33405 Talence Cedex, FRANCE \\ ochemelabri.fr
}

Raspaud and Sopena showed that the oriented chromatic number of a graph with acyclic chromatic number $k$ is at most $k 2^{k-1}$. We prove that this bound is tight for $k \geq 3$. We also show that some improper and/or acyclic colorings are NP-complete on a class $\mathcal{C}$ of planar graphs. We try to get the most restrictive conditions on the class $\mathcal{C}$, such as having large girth and small maximum degree. In particular, we obtain the NP-completeness of 3-ACYCLIC COLORABILITY on bipartite planar graphs with maximum degree 4, and of 4-ACYCLIC COLORABILITY on bipartite planar graphs with maximum degree 8.

Keywords: acyclic colorings, oriented colorings, NP-completeness

\section{Introduction}

Oriented graphs are directed graphs without opposite arcs. In other words an oriented graph is an orientation of an undirected graph, obtained by assigning to every edge one of the two possible orientations. If $G$ is a graph, $V(G)$ denotes its vertex set, $E(G)$ denotes its set of edges (or arcs if $G$ is an oriented graph). A homomorphism from an oriented graph $G$ to an oriented graph $H$ is a mapping $\varphi$ from $V(G)$ to $V(H)$ which preserves the arcs, that is $(x, y) \in E(G) \Longrightarrow(\varphi(x), \varphi(y)) \in E(H)$. We say that $H$ is a target graph of $G$ if there exists a homomorphism from $G$ to $H$. The oriented chromatic number $\chi_{o}(G)$ of an oriented graph $G$ is defined as the minimum order of a target graph of $G$. The oriented chromatic number $\chi_{o}(G)$ of an undirected graph $G$ is then defined as the maximum oriented chromatic number of its orientations. Finally, the oriented chromatic number $\chi_{o}(\mathcal{C})$ of a graph class $\mathcal{C}$ is the maximum of $\chi_{o}(G)$ taken over every graph $G \in \mathcal{C}$. We use in this paper the following notations:

$\mathcal{P}_{k}$ denotes the class of planar graphs with girth at least $k$.

$o c(k)$ denotes the class of graphs with oriented chromatic number at most $k$.

$\mathcal{S}_{k}$ denotes the class of graphs with maximum degree at most $k$.

$\mathcal{T}_{k}$ denotes the class of partial $k$-trees.

$\mathcal{D}_{k}$ denotes the class of $k$-degenerate graphs.

bip denotes the class of bipartite graphs.

\footnotetext{
${ }^{\dagger}$ Research supported in part by COMBSTRU.

1365-8050 (c) 2005 Discrete Mathematics and Theoretical Computer Science (DMTCS), Nancy, France
} 
A vertex coloring $c$ of a graph $G$ is acyclic if for every two distinct colors $i$ and $j$, the edges $u v$ such that $c(u)=i$ and $c(v)=j$ induce a forest. The acyclic chromatic number $\chi_{a}(G)$ is the minimum number of colors needed in an acyclic proper coloring of the graph $G$. Similarly, the acyclic chromatic number $\chi_{a}(\mathcal{C})$ of a graph class $\mathcal{C}$ is the maximum of $\chi_{a}(G)$ taken over every graph $G \in \mathcal{C}$. Raspaud and Sopena showed in [12] that:

Proposition 1 [12] For every graph $G$ such that $\chi_{a}(G)=k, \chi_{o}(G) \leq k 2^{k-1}$.

Together with the result of Borodin that planar graphs are acyclically 5-colorable (i.e. $\chi_{a}\left(\mathcal{P}_{3}\right)=5$ ), this implies that the oriented chromatic number of a planar graph is at most 80 (i.e. $\chi_{o}\left(\mathcal{P}_{3}\right) \leq 80$ ), which is yet the best known upper bound. See $[3,11]$ for the known upper bounds on $\chi_{o}\left(\mathcal{P}_{n}\right)$ for $n \geq 4$. In order to get a better upper bound on $\chi_{o}\left(\mathcal{P}_{3}\right)$, if possible, it is interesting to study the tightness of Proposition 1 , in particular for $k=5$. The previously best known lower bound on the maximum value of $\chi_{o}(G)$ in terms of $\chi_{a}(G)$ was given in [16] with a family of graphs $G_{k}, k \geq 1$ such that $\chi_{a}\left(G_{k}\right)=k$ and $\chi_{o}\left(G_{k}\right)=2^{k}-1$.

The notion of acyclic improper coloring was introduced in [1]. Let $\mathcal{C}_{0}, \ldots, \mathcal{C}_{k-1}$ be graph classes. A graph $G$ belongs to the class $\mathcal{C}_{0} \odot \cdots \odot \mathcal{C}_{k-1}$ if and only if $G$ has an acyclic $k$-coloring such that the $i^{t h}$ color class induces a graph in $\mathcal{C}_{i}$, for $0 \leq i \leq k-1$. The main motivation in the study of acyclic improper colorings is the following generalization of Proposition 1.

Proposition 2 [1] Let $\mathcal{C}_{0}, \ldots, \mathcal{C}_{k-1}$ be graph classes such that $\chi_{o}\left(\mathcal{C}_{i}\right)=n_{i}$, for $0 \leq i<k$. Every graph $G \in \mathcal{C}_{0} \odot \cdots \odot \mathcal{C}_{k-1}$ satisfies $\chi_{o}(G) \leq 2^{k-1} \sum_{i=0}^{i<k} n_{i}$.

The bound of Proposition 2 is shown to be tight for $k \geq 3$ under mild assumptions in Section 2 .

We know from $[13,15]$ that $\chi_{o}\left(\mathcal{T}_{3}\right)=\chi_{o}\left(\mathcal{T}_{3} \cap \mathcal{P}_{3}\right)=16$. Thus, Boiron et al. [1] point out that:

1. $\mathcal{P}_{3} \subset \mathcal{T}_{3} \odot \mathcal{S}_{0} \odot \mathcal{S}_{0}$ would imply that $\chi_{o}\left(\mathcal{P}_{3}\right) \leq 72$,

2. $\mathcal{P}_{3} \subset \mathcal{T}_{3} \odot \mathcal{S}_{1} \odot \mathcal{S}_{0}$ would imply that $\chi_{o}\left(\mathcal{P}_{3}\right) \leq 76$.

We will see that the second point is meaningless. Indeed, we have that $\mathcal{P}_{3} \subset \mathcal{T}_{3} \odot \mathcal{S}_{1} \odot \mathcal{S}_{0} \Longleftrightarrow \mathcal{P}_{3} \subset$ $\mathcal{T}_{3} \odot \mathcal{S}_{0} \odot \mathcal{S}_{0}$ from a general result on acyclic improper colorings of planar graphs given in Section 3.

In Section 4, we prove the NP-completeness of five coloring problems where the input graph is planar with some large girth and low maximum degree. ${ }^{\ddagger}$.

\section{Acyclic improper coloring versus oriented coloring}

Theorem 1 Let $k \geq 3$. Let $\mathcal{C}_{0}, \ldots, \mathcal{C}_{k-1}$ be hereditary graph classes closed under disjoint union, and such that $\chi_{o}\left(\mathcal{C}_{i}\right)=n_{i}$. Then $\chi_{o}\left(\mathcal{C}_{0} \odot \cdots \odot \mathcal{C}_{k-1}\right)=2^{k-1} \sum_{i=0}^{i<k} n_{i}$.

Proof: We construct an oriented graph $G \in \mathcal{C}_{0} \odot \cdots \odot \mathcal{C}_{k-1}$ such that $\chi_{o}(G)=2^{k-1} \sum_{i=0}^{i<k} n_{i}$. Let $u_{1}, u_{2}, u_{3}$ be a directed 2-path with arcs $u_{1} u_{2}$ and $u_{2} u_{3}$, or $u_{3} u_{2}$ and $u_{2} u_{1}$. We say that $u_{1}$ and $u_{3}$ are the endpoints of the directed 2-path. By definition, the endpoints of the directed 2-path get distinct colors in any oriented coloring. Since $\chi_{o}\left(\mathcal{C}_{i}\right)=n_{i}$, there exists a witness oriented graph $W^{i}$ such that $\chi_{o}\left(W^{i}\right)=n_{i}$. The graph $G_{i}$ contains $k-1$ independent vertices $v_{j}^{i}, 0 \leq j<k-1$ and $2^{k-1}$ disjoint

\footnotetext{
$\$$ A full paper version of this extended abstract containing all proofs is available at: http://dept-info.labri.fr/ ochem/acyclic.ps
} 
copies $W_{l}^{i}, 0 \leq l<2^{k-1}$ of $W^{i}$. We consider the binary representation $l=\sum_{n=0}^{n<k-1} 2^{n} x_{n}(l)$ of $l$. For every two vertices $v_{j}^{i}$ and $u_{l}^{i} \in W_{l}^{i}$, we put the $\operatorname{arc} v_{j}^{i} u_{l}^{i}$ (resp. $u_{l}^{i} v_{j}^{i}$ ) if $x_{j}(l)=1$ (resp. $x_{j}(l)=0$ ). If $l \neq l^{\prime}$, their binary representations differ at the $n^{t h}$ digit, thus $u_{l}^{i} \in W_{l}^{i} u_{l^{\prime}}^{i} \in W_{l^{\prime}}^{i}$ are the endpoints of a directed 2-path $u_{l}^{i}, v_{n}^{i}, u_{l^{\prime}}^{i}$. So the same color cannot be used in distinct copies of $W^{i}$, which means that at least $2^{k-1} n_{i}$ colors are needed to color the copies of $W^{i}$ in any oriented coloring of $G_{i}$. We acyclically color $G_{i}$ as follows. The $k-1$ vertices $v_{j}^{i}$ get pairwise distinct colors in $\{0, \ldots, k-1\} \backslash\{i\}$ and every vertex $u_{l}^{i}$ get color $i$ (that is why we need the "closed under disjoint union" assumption). Let $S_{i}$ denote the set of colors in some oriented coloring of the vertices $u_{l}^{i}$ of $G_{i}$. Now we take one copy of each graph $G_{i}$ and finish the construction of $G$. For every two vertices $u_{l}^{i} \in W_{l}^{i}$ and $u_{l^{\prime}}^{i^{\prime}} \in W_{l^{\prime}}^{i^{\prime}}$, such that $i \neq i^{\prime}$, we add a new vertex $l$ and create a directed 2-path $u_{l}^{i}, l, u_{l^{\prime}}^{i^{\prime}}$. So, for $i \neq i^{\prime}$, we have $S_{i} \cap S_{i^{\prime}}=\emptyset$, which means that at least $2^{k-1} \sum_{i=0}^{i<k} n_{i}$ colors are needed in any oriented coloring of $G$. To obtain an acyclic coloring of $G$, the new vertex $l$ adjacent to $u_{l}^{i}$ and $u_{l^{\prime}}^{i^{\prime}}$ gets a color in $\{0, \ldots, k-1\} \backslash\left\{i, i^{\prime}\right\}$, which is non-empty if $k \geq 3$.

Notice that Theorem 1 cannot be extended to the case $k=2$ in general. By setting $k=2$ and $\mathcal{C}_{0}=\mathcal{C}_{1}=\mathcal{S}_{0}$, we obtain the class of forests $\mathcal{S}_{0}^{(2)}=\mathcal{D}_{1}$. Proposition 2 provides the bound $\chi_{o}\left(\mathcal{S}_{0}^{(2)}\right) \leq 4$. This is not a tight bound, since oriented forests have a homomorphism to the oriented triangle, and thus $\chi_{o}\left(\mathcal{S}_{0}^{(2)}\right)=3$.

The proof of Theorem 1 is constructive, but it does not help for the problem of determining $\chi_{o}\left(\mathcal{P}_{3}\right)$. Indeed, the graph corresponding to the proper 5-acyclic coloring (i.e. $k=5$ and $\mathcal{C}_{0}=\cdots=\mathcal{C}_{4}=\mathcal{S}_{0}$ ) is highly non-planar (it contains e.g. $K_{32,48}$ as a minor).

\section{Acyclic improper colorings of planar graphs}

Theorem 2 Let $2 \leq k \leq 4$.

Then $\mathcal{P}_{3} \subset \mathcal{C}_{0} \odot \cdots \odot \mathcal{C}_{k-2} \odot o c(14) \Longleftrightarrow \mathcal{P}_{3} \subset \mathcal{C}_{0} \odot \cdots \odot \mathcal{C}_{k-2} \odot S_{0}$.

Theorem 2 allows us to study which statement of the form "every planar graph belongs to $\mathcal{C}_{0} \odot \cdots \odot \mathcal{C}_{k-1}$ " may improve the upper bound $\chi_{o}\left(\mathcal{P}_{3}\right) \leq 80$. If $k=4$, the "least" candidate class would be $o c(15) \odot S_{0} \odot$ $S_{0} \odot S_{0}$, but the corresponding bound is too large $\left(2^{4-1}(15+1+1+1)=144>80\right)$. If $k=1$, there must be exactly one improper color, otherwise the least candidate $o c(15) \odot o c(15) \odot S_{0}$ provides a too large bound $\left(2^{3-1}(15+15+1)=124>80\right)$.

The constant 14 in Theorem 2 can be improved to 15 using ideas in [15].

We have not been able to get similar results for planar graphs with larger girth.

\section{NP-complete colorings}

A graph $G$ belongs to the class $\mathcal{C}_{0} \circ \cdots \circ \mathcal{C}_{k-1}$ if and only if $G$ has a $k$-coloring such that the $i^{t h}$ color class induces a graph in $\mathcal{C}_{i}$, for $0 \leq i \leq k-1$. For brevity, if $\mathcal{C}_{0}=\cdots=\mathcal{C}_{k-1}=\mathcal{C}$ we will denote by $\mathcal{C}^{k}$ the class $\mathcal{C}_{0} \circ \cdots \circ \mathcal{C}_{k-1}$ and by $\mathcal{C}^{(k)}$ the class $\mathcal{C}_{0} \odot \cdots \odot \mathcal{C}_{k-1}$. If $\mathcal{C}_{1}$ and $\mathcal{C}_{2}$ are graph classes, we note $\left(\mathcal{C}_{1}: \mathcal{C}_{2}\right)$ the problem of deciding whether a given graph $G \in \mathcal{C}_{1}$ belongs to $\mathcal{C}_{2}$. If $P_{1}$ and $P_{2}$ are decision problems, we note $P_{1} \propto P_{2}$ if there is a polynomial reduction from $P_{1}$ to $P_{2}$.

Kratochvil proved that PLANAR $(3, \leq 4)$-SAT is NP-complete [9]. In this restricted version of SAT, the graph of incidences variable-clause of the input formula must be planar, every clause is a disjonction of 
exactly three literals, and every variable occurs in at most four clauses. A subcoloring is a partition the vertex set into disjoint cliques. The problem 2-SUBCOLORABILITY is NP-complete on triangle-free planar graphs with maximum degree 4 [5, 7]. Notice that on triangle-free graphs, a 2-subcoloring correspond to a vertex partition into two graphs with maximum degree 1 . Finally, the problem 3-COLORABILITY is shown to be NP-complete on planar graphs with maximum degree 4 in [6].

Theorem 3 PLAnAR $(3, \leq 4)$-SAT $\propto\left(\mathcal{P}_{6} \cap \mathcal{S}_{3}: \mathcal{S}_{0} \circ \mathcal{S}_{1}\right)$

Notice that on triangle-free graphs, the $\mathcal{S}_{0} \circ \mathcal{S}_{1}$ coloring correspond to the $(1,2)$-subcoloring defined in [10]. Theorem 3 improves a result in [10] stating that $\left(\mathcal{P}_{4} \cap \mathcal{S}_{3}: \mathcal{S}_{0} \circ \mathcal{S}_{1}\right)$ is NP-complete.

Theorem 4 PLANAR $(3, \leq 4)$-SAT $\propto\left(\mathcal{P}_{10} \cap \mathcal{S}_{3} \cap\right.$ bip $\left.: \mathcal{S}_{0} \odot \mathcal{S}_{1}\right)$

Theorem $5\left(\mathcal{P}_{4} \cap \mathcal{S}_{4}: \mathcal{S}_{1}^{2}\right) \propto\left(\mathcal{P}_{8} \cap \mathcal{S}_{4} \cap\right.$ bip : $\left.\mathcal{S}_{1}^{(2)}\right)$

Theorem $6\left(\mathcal{P}_{3} \cap \mathcal{S}_{4}: \mathcal{S}_{0}^{3}\right) \propto\left(\mathcal{P}_{4} \cap \mathcal{S}_{4} \cap\right.$ bip $\left.\cap \mathcal{D}_{2}: \mathcal{S}_{0}^{(3)}\right)$

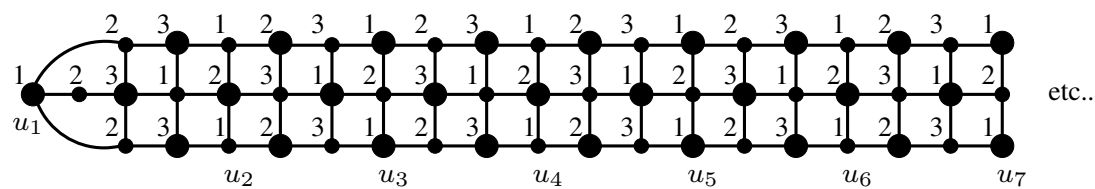

Figure 1: The vertex gadget for the reduction of Theorem 6 .

Given a planar graph $G$, we construct the graph $G^{\prime}$ as follows. We replace every vertex $v$ of $G$ by a copy of the vertex gadget depicted in Figure 1 and for every edge $v w$ we link a big vertex $u_{i}$ in the gadget of $v$ to a small vertex $u_{i}$ in the gadget of $w$. The given 3-acyclic coloring of the vertex gadget is the unique one up to permutation of colors. Notice that all $u_{i}$ get the same color and there exists no alternating path between distinct $u_{i}$. This common color in the gadget of a vertex $v$ correspond to the color of $v$ in a 3-coloring of $G$. Thus $G^{\prime}$ is acyclically 3-colorable if and only if $G$ is 3-colorable.

Theorem $7\left(\mathcal{P}_{3} \cap \mathcal{S}_{4}: \mathcal{S}_{0}^{3}\right) \propto\left(\mathcal{P}_{4} \cap \mathcal{S}_{8} \cap\right.$ bip $\left.\cap \mathcal{D}_{2}: \mathcal{S}_{0}^{(4)}\right)$

\section{References}

[1] P. Boiron, E. Sopena and L. Vignal. Acyclic improper colourings of graphs, J. Graph Theory 32 (1999), 97-107.

[2] O.V. Borodin. On acyclic colorings of planar graphs, Discrete Math. 25 (1979), 211-236.

[3] O.V. Borodin, A.V. Kostochka, J. Nešetřil, A. Raspaud and E. Sopena. On the maximum average degree and the oriented chromatic number of a graph, Discrete Math. 206 (1999), 77-89.

[4] O.V. Borodin, A.V. Kostochka and D.R. Woodall. Acyclic colorings of planar graphs with large girth, J. London Math. Soc. 60 (1999), 344-352.

[5] J. Fiala, K. Jansen, V.B. Le and E. Seidel. Graph Subcolorings: Complexity and Algorithm, SIAM J. Discrete Math. 16(4) (2003), 635-650. 
[6] M.R. Garey, D.S. Johnson and L.J. Stockmeyer. Some simplified NP-Complete graph problems, Theor. Comput. Sci. 1(3) (1976), 237-267.

[7] J. Gimbel and C. Hartman. Subcolorings and the subchromatic number of a graph, Discrete Math. 272 (2003), 139-154.

[8] A.V. Kostochka and L.S. Mel'nikov. Note to the paper of Grünbaum on acyclic colorings, Discrete Math. 14 (1976), 403-406.

[9] J. Kratochvil. A Special Planar Satisfiability Problem and a Consequence of its NP-Completeness, Discrete Appl. Math. 52 (1994), 233-252.

[10] H. Le and V.B. Le. The NP-completeness of $(1, r)$-subcolorability of cubic graphs, Inform. Process. Lett. 81(3) (2002), 157-162.

[11] P. Ochem. Oriented colorings of triangle-free planar graphs, Inform. Process. Lett. 92(2) (2004), 71-76.

[12] A. Raspaud and E. Sopena. Good and semi-strong colorings of oriented planar graphs, Inform. Process. Lett. 51 (1994), no.4, 171-174.

[13] E. Sopena. The chromatic number of oriented graphs, J. Graph Theory 25 (1997), 191-205.

[14] E. Sopena. Oriented graph coloring, Discrete Math. 229(1-3) (2001), 359-369.

[15] E. Sopena. There exist oriented planar graphs with oriented chromatic number at least sixteen, Inform. Process. Lett. 81(6) (2002), 309-312.

[16] L. Vignal. Homomorphismes et colorations de graphes orientés, Ph.D. Thesis, LaBRI, Université Bordeaux I (1997). 
